\title{
Dietary Supplement Use in Live Kidney Donors and Recipients
}

This article was published in the following Dove Press journal:

Transplant Research and Risk Management

\author{
Nicole Persun ${ }^{\prime}$ \\ David Johnson ${ }^{1,2}$ \\ Amanda Leonberg-Yoo ${ }^{2,3}$ \\ Jehan Bahrainwala ${ }^{2,3}$ \\ Peter P Reese ${ }^{2-4}$ \\ Brendan Steiner ${ }^{\prime}$ \\ Brooke Witmer' \\ Jamal Rashid' \\ Ali Naji iD ${ }^{2,5}$ \\ Jennifer Trofe-Clark (D) ${ }^{1-3}$ \\ 'Department of Pharmacy Services, \\ Hospital of the University of \\ Pennsylvania, Philadelphia, PA, USA; \\ ${ }^{2}$ Penn Medicine Transplant Institute, \\ Hospital of the University of \\ Pennsylvania, Philadelphia, PA, USA \\ ${ }^{3}$ Division of Renal Electrolyte and \\ Hypertension, Perelman School of \\ Medicine, University of Pennsylvania, \\ Philadelphia, PA, USA; ${ }^{4}$ Department of \\ Biostatistics, Epidemiology and \\ Informatics, Perelman School of \\ Medicine, University of Pennsylvania, \\ Philadelphia, PA, USA; ${ }^{5}$ Division of \\ Transplantation, Department of Surgery, \\ Perelman School of Medicine, University \\ of Pennsylvania, Philadelphia, PA, USA
}

Correspondence: Jennifer Trofe-Clark Department of Pharmacy Services, Hospital of the University of Pennsylvania, Ground Rhoads Building - Pharmacy Services, 3400 Spruce Street, Philadelphia,

PA 19104, USA

$\mathrm{Tel}+|2| 5-6 \mid 4-4274$

Fax $+1215-6 \mid 5-4900$

Email Jennifer.Trofe-

Clark@pennmedicine.upenn.edu
Background: Dietary supplement use in living kidney donors (LKDs) and kidney transplant recipients (KTRs) provides care challenges due to drug interactions, limited data, and lack of management guidelines. The study purposes were to describe supplement use patterns and intra/postoperative complications in these populations.

Methods: A single-center retrospective review of LKDs and KTRs from September 2016-2018 was conducted. Supplement information was collected at evaluation and preoperative visits. Supplement relationship to intra/postoperative complications was graded by Naranjo Adverse Drug Reaction Probability Scale.

Results: One hundred and fifty-seven LKDs met inclusion with an average age of 45 (SD 11) years. Thirty-seven LKDs (24\%) reported supplement use pre-donation. One hundred and eleven KTRs met inclusion with an average age of 46 (SD 13) years and 21 (19\%) reported supplement use pre-transplant. No significant differences in sex, ethnicity, or education were found between LKDs or KTRs using supplements vs none. One complication in an LKD was "possibly" related to supplement use vs none in KTRs.

Conclusion: Transplant providers should screen for supplement use in LKDs and KTRs at both the evaluation and pre-operative visits and review perioperative management. The low dietary supplement-related intra/post complication rate may be due to discontinuation of supplements preoperatively.

Keywords: dietary supplement, living kidney donation, kidney transplantation

\section{Introduction}

Dietary supplement use among US adults has significantly increased over the last decade. ${ }^{1}$ While many consumers buy these products over the counter may consider them harmless, their use does not come without the potential for side effects including hepatic or renal toxicities. ${ }^{2-4}$ A survey of 526 patients hospitalized for surgery found that $230(44 \%)$ were taking dietary supplements. Of those who reported supplement use, $17 \%$ were taking supplements that could potentially interact with anesthesia, and $10 \%$ were taking supplements that could potentially interact with antithrombotic drugs taken perioperatively. Three patients in the latter group were noted to experience intraoperative bleeding. ${ }^{2}$

The use of dietary supplements prior to living kidney donation and transplantation provides a challenge for providers, as no formal management guidelines exist. However, due to the reported risks noted above, our transplant center recommends discontinuation of the majority of dietary supplements at the time of the donor or recipient's initial evaluation by their living donor or transplant recipient nephrologist, respectively. Patient medication lists are also re-reviewed face to face by a 
transplant pharmacist during a second phase preoperative visit with the transplant team. This visit takes place approximately 1 week prior to surgery. While we recommend discontinuation of dietary supplements, not all patients comply and may continue using throughout donation or transplant, risking potential supplement-related intra/postoperative complications.

To the best of our knowledge, no literature exists on the use of dietary supplements in living kidney donors (LKDs) and kidney transplant recipients (KTRs) in the United States. We aimed to: (1) describe dietary supplement use patterns in LKDs and KTRs at a high volume, diverse transplant center in the United States, and intra/ postoperative complications in these populations and (2) raise awareness regarding the importance of screening for supplement use during the evaluation period for LKDs and KTRs and review perioperative management.

\section{Patients and Methods}

This was a retrospective, non-randomized, open-label review of non-HIV positive LKDs and KTRs who underwent surgery for donation or transplant at the Hospital of the University of Pennsylvania between September 30, 2016 and September 30, 2018. Patients positive for HIV infection were excluded from the analysis to meet our type of Institutional Review Board (IRB) application approval criteria. Informed consent to review patient medical records was not required, and a HIPAA waiver was granted by the IRB since the number of subjects would be prohibitive to contact and we do not still have contact with all patients; hence, some contact information would be outdated. The data were anonymized and maintained in a confidential manner consistent with our IRB approved practices and are in compliance with the Declaration of Helsinki.

Dietary supplements are defined by the Dietary Supplement Health and Education Act (DSHEA) as a product (other than tobacco) intended to supplement the diet, that contains one or more of the following dietary ingredients: vitamins, minerals, herb or botanical, amino acids, a dietary substance for use by man to supplement the diet by increasing the total dietary intake, a concentrate, metabolite, constituent, extract, or combination of any ingredient..$^{5}$ For the purposes of this study, dietary supplement use was defined as orally administered herbal or complementary nutritional products (including botanicals, microbial additives, and amino acids), excluding micronutrients (vitamins/minerals) and food/drinks. Micronutrients were excluded since it was not clear in this retrospective analysis if these were prescribed by a provider for a specific indication.

The primary purpose of this study was to describe dietary supplement use patterns in the pre-donation/pretransplant setting. Supplement use was documented in the chart at the initial evaluation visit by a donor or transplant nephrologist, respectively, and at the preoperative visit by a transplant pharmacist. Additionally, we aimed to describe any documented dietary supplement-related complications that occur during donation or transplant surgery admission and relationship of intra/post complication(s) with dietary supplement use graded by the Naranjo Adverse Drug Reaction (ADR)Probability Scale. ${ }^{6}$

Patient demographic information was collected via the electronic health record at the time of evaluation and included baseline characteristics (age, reported sex, ethnicity, reported education beyond high school, and relationship of the donor to the recipient). Date of evaluation, preoperative visit and donation/transplant were collected. Type, dose, and number of dietary supplements documented were collected for the evaluation visit, preoperative visit, and donation/transplant admission. Information regarding all complications documented within the donation/transplant admission encounter was also collected from chart review of the surgical case and/or discharge documentation. This included any requirement of intraoperative blood transfusion and potential delays in hospital admission discharge.

Descriptive statistics were performed using Microsoft Excel. Categorical variables were analyzed by Fisher's exact test performed using Graph Pad Quick Calcs Prism version 8.0. A two-sided $P$ - value of less than 0.05 was considered statistically significant. Relationship of complication related to dietary supplement use was graded by the Naranjo ADR Probability Scale and classified as definite, probable, possible, or doubtful ADRs. ${ }^{6}$

\section{Results}

One hundred and fifty-seven LKDs met inclusion with an average age of 45 (SD 11) years. Thirty-seven (24\%) LKDs reported dietary supplement use at any time prior to donation, while 120 LKDs had no chart-documented report of dietary supplement use. No statistically significant differences were identified in dietary supplement use by age, sex, ethnicity, or education status when comparing LKDs who reported use of supplements to LKDs that did not, though numerically, a higher percent of those who used supplements were $\geq 40$ years old, of male sex, of nonwhite ethnicity and had reported formal education beyond 
Table I Demographics of Living Kidney Donors and Recipients

\begin{tabular}{|l|l|l|l|}
\hline (A) Characteristics of Living Kidney Donors (LKDs) & No Dietary Supplement (n= I 20) & Dietary Supplement ${ }^{\dagger}$, (n=37) $^{\prime}$ & $\boldsymbol{P}_{\text {-value }}$ \\
\hline Characteristic & $76(63)$ & $28(76)$ & 0.23 \\
\hline Age $\geq 40$ years (\%) & $47(39)$ & $15(4 I)$ & 1 \\
\hline Reported Male sex (\%) & & & $30(8 I)$ \\
\hline $\begin{array}{l}\text { Reported ethnicity, } \\
\text { white (\%) } \\
\text { non-white (\%) }\end{array}$ & $101(84)$ & $7(19)$ & 0.62 \\
\hline Reported formal education beyond high school (\%) & $89(74)$ & $29(78)$ & 0.67 \\
\hline
\end{tabular}

(B) Characteristics of Recipients of Living Donor Kidneys (KTRs)

\begin{tabular}{|c|c|c|c|}
\hline Characteristic & No Dietary Supplement $(n=90)$ & Dietary Supplement ${ }^{\ddagger},(n=2 I)$ & $P$-value \\
\hline Age $\geq 40$ years $(\%)$ & $64(7 I)$ & $17(8 \mid)$ & 0.43 \\
\hline Reported Male sex (\%) & $50(56)$ & II (52) & 0.81 \\
\hline $\begin{array}{l}\text { Reported ethnicity, } \\
\text { white (\%) } \\
\text { non-white (\%) }\end{array}$ & $\begin{array}{l}72(80) \\
18(20)\end{array}$ & $\begin{array}{l}13(62) \\
8(38)\end{array}$ & 0.09 \\
\hline Reported formal education beyond high school (\%) & $59(66)$ & $18(86)$ & 0.11 \\
\hline
\end{tabular}

Notes: †Dietary supplement use at any time prior to donation. ${ }^{\ddagger}$ Dietary supplement use at any time prior to transplant. For the purposes of this study, dietary supplement use was defined as orally administered herbal or complementary nutritional products (including botanicals, microbial additives, and amino acids), excluding micronutrients (vitamins/minerals), and foods/drinks.

high school (Table 1A). Twenty-one LKDs (13\%) reported taking a dietary supplement at the first evaluation visit. The presence of dietary supplement use was not recorded for 76 LKDs $(48 \%)$ during the initial evaluation. Twentyfour LKDs (15\%) reported dietary supplement use at their pre-operative visit, $63 \%$ of which were first identified at this visit. The average time between the pre-operative visit and donation was 9 (SD 5) days. The most commonly reported dietary supplements at both the initial evaluation and pre-operative visits were (1) omega-3-fatty acids, (2) glucosamine/chondroitin or probiotics (3) lysine. Of the patients that reported dietary supplements, $41 \%$ reported taking more than one supplement, and each of these patients was at least 40 years old.

Six $(16 \%)$ chart-documented complications were noted in the LKD supplement group versus 23 (19\%) chart-document complications in the non-supplement group (Figure 1A). In the supplement group, the relationship of each complication with a dietary supplement was graded by the Naranjo ADR Probability Scale. ${ }^{6}$ One (17\%) cardiovascular complication was classified as a possible ADR related to black cohosh, which was noted to be an active medication at transplant admission, despite the patient being instructed to discontinue pre-surgery. Five other complications $(83 \%)$ were classified as doubtful ADRs. No excessive intraoperative bleeding could be confirmed as there were no documented intraoperative blood transfusions. The average length of stay (LOS) was 3 days for all LKDs, with no difference in LOS for those patients with complications.

Only $111 / 217$ (51\%) KTRs had a preoperative visit encounter documented in the chart as completed by a pharmacist during the study period, and therefore 106 were excluded. Of the included KTRs, the average age was 46 (SD 13) years. Twenty-one (19\%) KTRs reported dietary supplement use at any time prior to transplant, while 90 KTRs had no chart-documented report of dietary supplement use. Similar to the LKD group, there were no statistically significant differences between KTRs that reported supplement use versus those that did not, though numerically, a higher percent of those who used supplements were $\geq 40$ years old, were of non-white ethnicity and had reported formal education beyond high school. Seven (6\%) KTRs had chart-documented dietary supplement use at the evaluation visit and $20(18 \%)$ at the preoperative visit, which were documented by the transplant pharmacist. The most commonly reported dietary supplements were (1) omega-3-fatty acids, (2) probiotics, (3) coenzyme Q-10. 


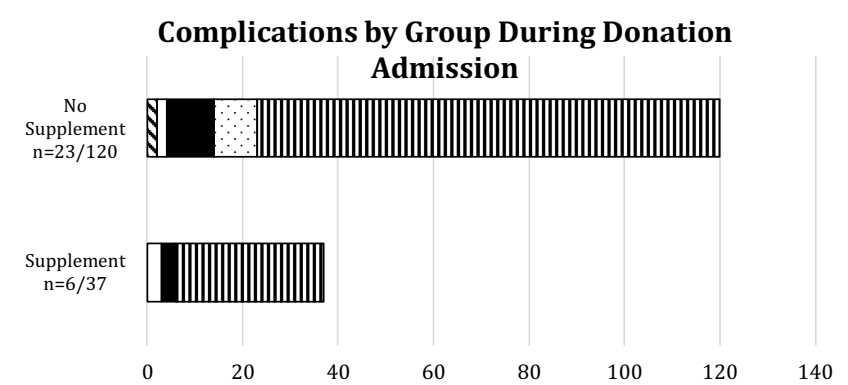

Complications by Group During Donation

No
Supplement

$\mathrm{n}=23 / 120$

20

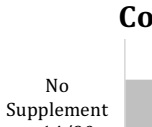

Complications by Group During Transplant

Admission

$n=14 / 90$

Supplement

$n=2 / 21$

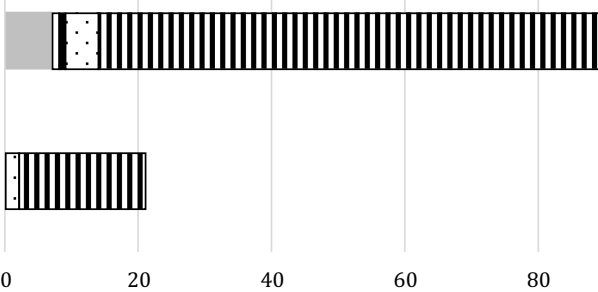

020
100

Figure I Depiction of all chart-documented postoperative complications categorized by dietary supplement use for donors (A) and recipients (B). (A) Chart-documented Postoperative Live Kidney Donor Complications. (B) Chart-documented Postoperative Live Kidney Donor Recipient Complications.

Notes: : Bleeding; $\mathbb{N}$ : Rhabdomyolysis; $\square$ : Cardiovascular; $\square$ : Infection; $\square$ : *Other; $\left[\mathbf{l l l l}\right.$ : No Complication. ${ }^{*}$ Other: Urinary retention, respiratory complications, hypocalcemia. No rheumatologic complications were noted. For the purposes of this study, dietary supplement use was defined as orally administered herbal or complementary nutritional products (including botanicals, microbial additives, and amino acids), excluding micronutrients (vitamins/minerals), and foods/drinks.

Two (10\%) chart-documented complications were noted in the KTR supplement group versus 14 (16\%) chart-document complications in the non-supplement group (Figure 1B). Both complications in the supplement group were classified as doubtful ADRs. The average length of stay (LOS) was 4 days for all KTRs, with no difference in LOS for those with complications.

Five KTRs received a kidney from their spouse within the LKD group. Upon review, 4/5 of those KTRs had chart-documented dietary supplement use prior to transplant. In contrast, only one LKD spouse had chart-documented dietary supplement use. This LKD had been using lysine and cranberry extract while the KTR spouse had been using omega-3 fatty acids and yohimbe.

\section{Discussion}

The popularity of dietary supplements has continued to increase over the years as an effort to manage chronic illnesses and as a strategy to promote overall health and well-being. The prevalence of dietary supplement use in our population (19-24\%) was found to be slightly lower than the US national average $(>40 \%){ }^{1}$

Particular characteristic traits have been studied and associated with dietary supplement use. National data found that age greater than 70 years old, education beyond high school, use of prescription or over the counter medications and presence of a chronic disease state were all factors related to dietary supplement use. ${ }^{1}$ Consistent with these data, the majority of LKDs and KTRs that reported dietary supplement use in our study were older, of white ethnicity and had formal education beyond high school. However, these findings were not significantly different than those patients not taking dietary supplements, and are similar to national transplant kidney data. ${ }^{7}$

There may be a misconception that dietary supplements are just as safe, effective and represent similar quality to prescription medications. Dietary supplements are placed under the Dietary Supplement Health and Education Act (DSHEA) as a food rather than a drug. ${ }^{5}$ Therefore, practices surrounding their manufacturing and development continue to be a concern. ${ }^{4}$ Additionally, some supplements may incur nephrotoxicity with their use, especially in the setting of end-stage renal disease or may have the potential to interact with anesthesia or perioperative antithrombotic therapies., ${ }^{2,3}$ Fortunately, a low number of complications were found in our overall study population and only one complication was identified as a possible ADR related to dietary supplement use via Naranjo Scale. ${ }^{6}$ This may be owed to the fact that a transplant provider saw patients twice during the evaluation phase and on average at least 1 week prior to surgery, thus, allowing enough time to discontinue any supplement which may induce complications. Furthermore, it is important to note that the Naranjo scale provides information on the probability of a drug to cause such an adverse effect related to the reported complications, realizing that causes of intra/ postoperative complications are often multifactorial.

Many of the dietary supplements identified at face-to-face pre-operative visits with the transplant pharmacist were not previously identified or recorded by other providers during initial evaluations. During the transplant pharmacy visit, patients are asked not only about prescription medications but also if they may be taking any over the counter, or dietary supplements on a regular basis. It is known that patients often under-disclose the use of dietary supplements, which can be detrimental in the surgical setting. ${ }^{2,3}$ This finding suggests that 
transplant clinicians should vigilantly discuss dietary supplement use openly with our patients to provide the most appropriate and safe recommendations.

Several limitations exist within this study, including the retrospective design and single-center patient population. (1) Providers used a variable evaluation and preoperative visit template that did not consistently include questioning about dietary supplements. (2) Documentation was not consistent in the medical record in regards to whether supplements were selfinitiated or prescribed. (3) When questioned regarding dietary supplement use, documentation of administration may not have taken place if the provider immediately discontinued such medications during the visit. The rate of dietary supplement use disclosure may, therefore, be underreported in our study population. (4) Additionally, transplant pharmacist practices evolved during this period too. Current practice at our center is for a transplant pharmacist to have a face-to-face encounter at the preoperative visit with all LKDs and KTRs.

This study provides insight on the use of dietary supplements in LKDs and KTRs pre-surgery, a topic not yet reported on. We found that approximately $20 \%$ of LKDs and KTRs had used a dietary supplement within either the evaluation or preoperative phases. Patients at our center are typically instructed to discontinue dietary supplements 2-3 weeks prior to surgery. We did, however, find that a few patients had continued on dietary supplements or were not stopped until the preoperative visit, which was on average 9 days prior to donation surgery or 11 days prior to transplant. Intra/postoperative complications in those patients that had used dietary supplements were graded as unlikely to be caused by ADRs, using the Naranjo scale. ${ }^{6}$ Detection of intra/post-operative complications in patients who used dietary supplements prior to surgery was minimal. However, implementation of a thorough medication review process prior to surgery, with recommendations for discontinuation at least a week prior to surgery, may have decreased the risk of potential complications in this population. Providers should continue to have open discussions with all LKDs and KTRs regarding dietary supplement use due to concerns regarding safety, efficacy and adverse effects with dietary supplements. ${ }^{1-4}$ All LKDs and KTRs should be instructed to stop dietary supplements at least 1 week prior to surgery and to not resume use after donation or transplant unless first discussed with the donor/transplant team. Future prospective research in large LKD and KTR populations is needed to better define dietary supplement risks and potential impacts on evaluation, donation and transplant-related outcomes.

\section{Acknowledgments}

We are grateful to our kidney living donor coordinators: Donna Collins, RN, MSN, CTCC and Mary Houston, BSN, RN, CCTC, Stephanie M Neal, BS, BSN, RN and our pre-transplant recipient coordinators: Cynthia Gardiner, BSN, RN, Maral Palanjian RN, Rita R. Perry, BSN, RN, Meera Ragoopath, MPH, BSN, RN, Allyson Semple, BSN, RN, Tawny Uemura, BSN, RN for providing education about herbal supplements to donors and recipients in the evaluation phase of transplant care.

\section{Disclosure}

Nicole Persun now practices at Allegheny Health Network, Pittsburgh, PA, USA. David Johnson now practices at Alexion Pharmaceuticals Inc, Boston, MA, USA. David Johnson is currently an employee of Alexion Pharmaceuticals and the work contained within this manuscript is independent of Alexion. Peter P Reese reports (1) investigator-initiated grants from Merck and AbbVie to the University of Pennsylvania to support research on transplantation of $\mathrm{HCV}$-infected organs into uninfected recipients, followed by antiviral treatment; (2) investigator-initiated grants from CVS Caremark and Merck to the University of Pennsylvania to support research on medication adherence (focus: Statins); (3) consulting with COllaborative Healthcare Research \& Data Analytics (COHRDATA) on epidemiology of medications to improve laboratory parameters including potassium among dialysis patients; (4) associate editor for the American Journal of Kidney Diseases. The authors report no other conflicts of interest in this work.

\section{References}

1. Rashrash M, Schommer JC, Brown LM. Prevalence and predictors of herbal medicine use among adults in the United States. J Pt Exp. 2017;4:108-113.

2. Levy I, Attias S, Ben-Arye E, et al. Perioperative risks of dietary and herbal supplements. World J Surg. 2017;41:927-934. doi:10.1007/ s00268-016-3825-2

3. Gabardi S, Munz K, Ulbrich C. A review of dietary supplementinduced renal dysfunction. Clin J Am Soc Nephrol. 2007;2:757-765. doi:10.2215/CJN.00500107

4. Geller AI, Shehab N, Weidle NJ, et al. Emergency department visits for adverse events related to dietary supplements. NEJM. 2015;373:15311540. doi:10.1056/NEJMsa1504267

5. Dietary and Supplement Health and Education Act of 1994. Available from: https://ods.od.nih.gov/About/DSHEA_Wording.aspx. Accessed July 19, 2019.

6. Naranjo CA, Busto U, Sellers EM, et al. A method for estimating the probability of adverse drug reactions. Clin Pharmacol Ther. 1981;30:239-245. doi:10.1038/clpt.1981.154

7. Hart A, Smith JM, Skeans MA, et al. OPTN/SRTR 2017 annual data report: kidney. Am J Transplant. 2019;2:19-123. doi:10.1111/ajt.15274 


\section{Publish your work in this journal}

Transplant Research and Risk Management is an international, peer-reviewed open access journal focusing on all aspects of transplantation and risk management to achieve optimal outcomes in the recipient improving survival and quality of life. The manuscript management system is completely online and includes a very quick and fair peer-review system, which is all easy to use. Visit http:// www.dovepress.com/testimonials.php to read real quotes from published authors. 